\title{
Evaluation of the QT Interval in Patients with Drug-induced QT Prolongation and Torsades de Pointes
}

Philipp Krisai ${ }^{1}$, Konstantinos Vlachos ${ }^{2}$, F. Daniel Ramirez ${ }^{3}$, Yosuke Nakatani ${ }^{4}$, Takashi Nakashima ${ }^{5}$, Takamitsu Takagi ${ }^{6}$, Tsukasa Kamakura ${ }^{7}$, Elodie Surget ${ }^{1}$, Clémentine André $^{8}$, Ghassen Cheniti ${ }^{9}$, Nicolas Welte ${ }^{10}$, Remi Chauvel ${ }^{10}$, Romain Tixier $^{1}$, Josselin Duchateau ${ }^{11}$, Thomas Pambrun ${ }^{12}$, Nicolas Derval ${ }^{1}$, Mélèze Hocini ${ }^{13}$, Pierre Jais ${ }^{1}$, Michel Haissaguerre ${ }^{14}$, and Frederic Sacher ${ }^{15}$

${ }^{1}$ Centre Hospitalier Universitaire de Bordeaux

${ }^{2}$ Evangelismos General Hospital of Athens

${ }^{3}$ Centre Hospitalier Universitaire de Bordeaux Hopital Cardiologique

${ }^{4}$ University of Toyama

${ }^{5} 1$. Electrophysiology and Ablation Unit and L'Institut de rythmologie et modélisation cardiaque (LIRYC)

${ }^{6}$ Yokosuka Kyosai Hospital

${ }^{7}$ National Cerebral and Cardiovascular Center

${ }^{8} \mathrm{CHU}$ Trousseau

${ }^{9}$ Hôpital Cardiologique du Haut Lévêque

${ }^{10} \mathrm{CHU}$ Bordeaux, Hopital Haut Leveque

${ }^{11}$ IHU Liryc, Electrophysiology and Heart Modeling Institute, Fondation Bordeaux

Université, F-/ Bordeaux University Hospital (CHU), Electrophysiology and Ablation Unit

/ Univ. Bordeaux, Centre de recherche Cardio-Thoracique de Bordeaux, U1045

${ }^{12}$ Hôpital Cardiologique du Haut-Lévêque

${ }^{13}$ Hôpital Cardiologique du Haut-Lévèque

${ }^{14}$ Hopital Cardiologique du Haut-Leveque

${ }^{15}$ Bordeaux University Hospital

May 11, 2020

\footnotetext{
Abstract

Background Data on the optimal location of the ECG leads for the diagnosis of drug-induced long QT syndrome (diLQTS) with Torsades de Pointes (TdP) are lacking. Methods We systematically reviewed the literature for ECGs of patients with diLQTS and subsequent TdP. We assessed T-wave morphology in each lead and measured the longest QT interval in the limb and chest leads in a standardized fashion. Results Of 84 patients, $61.9 \%$ were female and mean age was 58.8 years. QTc was significantly longer in chest versus limb leads (mean (standard deviation) 671 (102) vs 655 (97) ms, p=0.02). Using only limb leads for QT interpretation, 18 (21.4\%) ECGs were non-interpretable: 10 (11.9\%) due to too flat T-waves, 7 (8.3\%) due to frequent, early PVCs and $1(1.2 \%)$ due to too low ECG recording quality. In the chest leads, ECGs were non-interpretable in $9(10.7 \%)$ patients: $6(7.1 \%)$ due to frequent, early PVCs, 1 (1.2\%) due to insufficient ECG quality, 2 (2.4\%) due to missing chest leads but none due to too flat T-waves. The most common T-wave morphologies in the limb leads were flat (51.0\%), broad $(14.3 \%)$ and late peaking (12.6\%) T-waves. Corresponding chest lead morphologies were inverted (35.5\%), flat (19.6\%) and biphasic (15.2\%) T-waves. Conclusions Our results indicate that QT evaluation by limb leads only underestimates the
} 
incidence of diLQTS experiencing TdP and favors the screening using both limb and chest lead ECG.

\section{Evaluation of the QT Interval in Patients with Drug-induced QT Prolongation and Torsades de Pointes}

Philipp Krisai $\mathrm{MD}^{1}$, Konstantinos Vlachos $\mathrm{MD}^{1}$, F. Daniel Ramirez MD MSc${ }^{1}$, Yosuke Nakatani MD ${ }^{1}$, Takashi Nakashima $\mathrm{MD}^{1}$, Takamitsu Takagi MD ${ }^{1}$, Tsukasa Kamakura $\mathrm{MD}^{1}$, Elodie Surget $\mathrm{MD}^{1}$, Clémentine André $\mathrm{MD}^{1}$, Ghassen Cheniti $\mathrm{MD}^{1}$, Nicolas Welte $\mathrm{MD}^{1}$, Rémi Chauvel $\mathrm{MD}^{1}$, Romain Tixier MD $\mathrm{MSc}^{1}$, Josselin Duchateau MD PhD ${ }^{1}$, Thomas Pambrun $\mathrm{MD}^{1}$, Nicolas Derval $\mathrm{MD}^{1}$, Mélèze Hocini $\mathrm{MD}^{1}$, Jaïs Pierre $\mathrm{MD}^{1}$, Haïssaguerre Michel $\mathrm{MD}^{1}$, Frédéric Sacher MD $\mathrm{PhD}^{1}$

Electrophysiology and Ablation Unit and L'Institut de rythmologie et modélisation cardiaque (LIRYC), Centre hospitalier universitaire de Bordeaux, Bordeaux-Pessac, France

Brief title: QT Interval in Drug-induced QT Prolongation

Word count (Text): 1632

Address for correspondence: Philipp Krisai MD Electrophysiology and Ablation Unit

Hospital Haut-Lévêque, Centre hospitalier universitaire de Bordeaux

Av. Magellan, 33600 Pessac, France Phone: +43 69917227228 E-mail: p.krisai@gmail.com

\section{Funding and disclosures}

Philipp Krisai is supported by the University of Basel, the Mach-Gaensslen foundation and the BangerterRhyner foundation. All other authors have nothing to disclose.

\section{Abstract \\ Background}

Data on the optimal location of the ECG leads for the diagnosis of drug-induced long QT syndrome (diLQTS) with Torsades de Pointes (TdP) are lacking.

\section{Methods}

We systematically reviewed the literature for ECGs of patients with diLQTS and subsequent TdP. We assessed T-wave morphology in each lead and measured the longest QT interval in the limb and chest leads in a standardized fashion.

\section{Results}

Of 84 patients, $61.9 \%$ were female and mean age was 58.8 years. QTc was significantly longer in chest versus limb leads (mean (standard deviation) 671 (102) vs 655 (97) $\mathrm{ms}, \mathrm{p}=0.02$ ). Using only limb leads for QT interpretation, 18 (21.4\%) ECGs were non-interpretable: $10(11.9 \%)$ due to too flat T-waves, 7 (8.3\%) due to frequent, early PVCs and $1(1.2 \%)$ due to too low ECG recording quality. In the chest leads, ECGs were non-interpretable in $9(10.7 \%)$ patients: $6(7.1 \%)$ due to frequent, early PVCs, $1(1.2 \%)$ due to insufficient ECG quality, $2(2.4 \%)$ due to missing chest leads but none due to too flat T-waves. The most common T-wave morphologies in the limb leads were flat (51.0\%), broad (14.3\%) and late peaking (12.6\%) T-waves. Corresponding chest lead morphologies were inverted (35.5\%), flat (19.6\%) and biphasic (15.2\%) T-waves.

\section{Conclusions}

Our results indicate that QT evaluation by limb leads only underestimates the incidence of diLQTS experiencing TdP and favors the screening using both limb and chest lead ECG.

Key words: Drug-induced long QT syndrome; Torsades de Pointes; ECG; screening.

\section{Introduction}


Drug-induced prolongation of the QT interval (diLQTS) substantially increases the risk for Torsades de Pointes (TdP) and sudden death. ${ }^{1}$ Offending agents include widely used drugs like antibiotics, antimalarials, antifungals, antivirals, anti-arrhythmic drugs (AAD), psychiatric drugs, and many others. ${ }^{1-3}$ In the current context of severe acute respiratory syndrome coronavirus 2 (SARS-CoV2) infections, ${ }^{4}$ drugs with a risk for diLQTS, like hydroxychloroquine, azithromycin or antiviral drugs, are used widely off-label and are investigated in randomized trials in large patient populations. ${ }^{5}$ Expert consensus statements therefore recommend ECG screening for QT interval prolongation before treatment initiation and regularly during treatment with these drugs. ${ }^{6,7}$ However, repeated ECG screening using 12-lead ECG is clinically unfeasible in the large number of isolated, hospitalized or ambulatory patients, may increase the risk for virus transmission and is economically unfeasible in low-income countries.

Mobile ECG devices seem to be able to reconstruct the six ECG limb leads with good reproducibility of basic ECG intervals compared to conventional 12-lead ECGs in patients with normal and prolonged QT interval. $^{8-11}$ They might therefore offer a lower-cost, clinically more convenient and safer screening modality for QT prolongation in diLQTS used by the patients themselves. However, as T-wave morphology in the limb leads may flatten in very long QT intervals with the highest risk for TdP, the diagnostic accuracy and interpretability of the QT interval might be low if only limb leads are used. ${ }^{12}$ Currently, systematic data on the diagnostic value of the limb versus chest leads for prolonged QT interval and T-wave morphology in patients with diLQTS and subsequent TdP are lacking.

We therefore aimed to systematically investigate the diagnostic value and interpretability of the limb versus the chest ECG leads in patients with diLQTS, who subsequently experienced TdP.

\section{Methods}

\section{Patient population}

We systematically reviewed the available literature for ECGs of patients with diLQTS and subsequent TdP. We entered the MeSH term 'drug induced long qt torsades de pointes' into Medline and Embase and identified 1253 reports published until April 19 ${ }^{\text {th }}, 2020$. Eligible articles had to include human subjects with available limb ECG leads with drug-induced QT interval prolongation and experiencing subsequent TdP. We also screened the references of eligible papers. After manual review, we identified 77 ECGs that were included in the current study. We also reviewed eligible ECGs with the same criteria from the hospital files of our institution (Hospital Haut-Leveque, University Hospital Bordeaux, Pessac, France) and furthermore included 7 patients.

\section{Study variables}

Patient demographics, medical history and medication were extracted as available. The ECGs were evaluated independently by cardiac electrophysiologists in a standardized manner. Disagreement was resolved by consensus. ECG interpretation included identification, interpretability and measurement of the longest QT interval in limb and chest leads, respectively, and the evaluation of the T-wave morphology in each available lead. T-wave morphology was categorized into flat, broad, late peaking, notched, biphasic or inverted. We then added up all limb and chest lead morphologies in order to evaluate the respective dominant morphology. For measurments, we used electronic calipers which we calibrated individually to each ECG for the QT interval, QRS duration and RR interval. The corrected QT (QTc) interval was calculated using Bazett's formula $\mathrm{QTc}=\mathrm{QT} /[?] \mathrm{RR}$ if heart rate was [?]90 beats per minute (bpm) and Fridericia's formula QTc= $\mathrm{QT} /\left(\mathrm{RR}^{\wedge} 0.33\right)$ if heart rate was $>90 \mathrm{bpm}$.

\section{Statistical analysis}

The distribution of continuous variables was checked by visual inspection of the histogram and by assessing skewness and kurtosis. As all variables were normally distributed, they were presented as means (+- standard deviations (SD)) and compared using paired Student's t-tests. We used Bland Altmann plots to investigate differences in QTc between limb and chest leads. A two-sided p-value $<0.05$ was considered statistically 
significant. All statistical analyses were performed using SAS 9.4 (SAS Corporation, Cary, North Carolina, USA).

\section{Results}

84 patients met the eligibility criteria. All patients experienced TdP and in 49 (58.3\%) patients early PVCs were present. Mean (SD) age was 58.8 (8.8) years and $52(61.9 \%)$ were female. Prior medical history included hypertension in 21 (25.0\%), ischemic heart disease in $13(15.5 \%)$, heart failure in $12(14.3 \%)$, atrial fibrillation in $8(9.5 \%)$ and renal impairment in $11(13.1 \%)$ patients. Figure 1 shows the medications that were suspected to cause QT interval prolongation. The three most common offending drug classes were anti-arrhythmic drugs, psychiatric drugs and antibiotics. Amiodarone $(\mathrm{n}=10)$, Methadone $(\mathrm{n}=9)$ and Dofetilide $(\mathrm{n}=7)$ were the most frequent individual drugs. In 26 (31.0\%) cases, patients took a combination of potentially QT interval prolonging drugs.

Mean (SD) QRS duration was 100 (26) ms and the mean (SD) heart rate was 66 (20) bpm. Figure 2 shows the Bland Altmann plot with a wide variation in the QTc differences between the limb and chest leads. Comparing the limb and chest leads, mean (SD) QT durations were 645 (129) and 661 (125) ms ( $\mathrm{p}=0.03$ ) and mean (SD) QTc durations were $655(97)$ and $671(102) \mathrm{ms}(\mathrm{p}=0.02)$, respectively. Using only the limb leads for QT interval interpretation, 18 (21.4\%) ECGs were non-interpretable: $10(11.9 \%)$ due to too flat T-waves, $7(8.3 \%)$ due to frequent, early PVCs and $1(1.2 \%)$ due to insufficient ECG recording quality. In the chest leads, it was not possible to interprete the QT interval in $9(10.7 \%)$ patients: $6(7.1 \%)$ due to frequent, early PVCs, 1 (1.2\%) due to insufficient ECG quality, 2 (2.4\%) due to missing chest leads but none due to too flat T-waves. Single limb and chest leads that were judged most often as the best for QT interval interpretation were II and V5, respectively.

Figure 3 shows detailed data on T-wave morphology for each individual lead and cumulative for limb and chest leads. The most common, cumulative T-wave morphologies in the limb leads were flat T-waves in $51.0 \%$, broad $\mathrm{T}$-waves in $14.3 \%$ and late peaking $\mathrm{T}$-waves in $12.6 \%$. The most common, cumulative morphologies in the chest leads were inverted T-waves in $35.5 \%$, flat T-waves in $19.6 \%$ and biphasic T-waves in $15.2 \%$. T-wave alternans was present in $6(7.1 \%)$ patients. Beside a high variability in T-wave morphology between patients, there was also high variability over the individual leads within the same patient as showcased in Figure 4.

\section{Discussion}

The current study in 84 patients with drug-induced QT interval prolongation and Torsades de Pointes showed lower diagnostic interpretability and shorter measured QT intervals in the limb leads in comparison to chest leads due to flattened T-waves. To the best of our knowledge, this is the largest study in this patient population to date.

We found that the QT interval was not reliably interpretable due to too flat T-waves in $11.9 \%$ of the patients with diLQTS with subsequent TdP, if only the limb leads were used. In comparison, the T-wave morphology in the chest leads was never a reason for non-interpretability of the QT interval. Therefore, approximately one out of nine patients with diLQTS and TdP might have been missed if only limb leads were used. Moreover QT duration was measured shorter in the limb leads compared to the chest leads though there was a high variability in the differences. Our results point out a possible limitation of QT interval monitoring in diLQTS with limb leads only, as for example by mobile ECG devices.

Prior studies on QT interval interpretation with mobile devices have mainly focused on the measurement of the QT interval and not on T-wave morphology. Garabelli et al. showed good agreement of the QTc interval between two limb leads by a mobile device compared to a 12-lead ECG in 99 healthy volunteers and patients loaded with dofetilide or sotalol up to a QT duration of $500 \mathrm{~ms}$, but with decreasing agreement above 500ms. ${ }^{9}$ Castelletti et al. investigated 351 measurements in 20 LQTS patients and 16 controls and also found good overall agreement between a single lead mobile ECG device and a 12-lead ECG. However, the range of disagreement also increased with increasing QTc duration. ${ }^{8}$ Moreover, two other studies in 381 and 
94 subjects without known QT interval prolongation, respectively, showed relevant discrepancies in the QT interval between single lead mobile ECG devices and 12-lead ECGs. ${ }^{13,14}$ Malone et al. furthermore reported that the QT interval was not measureable in $9 \%$ of their study subjects due to low T-wave amplitudes. ${ }^{10}$ Our results expand the current knowledge and suggest, that the interpretability of the limb leads further decreases with even longer QT intervals in patients experiencing TdP.

For optimal patient selection for mobile ECG monitoring, a screening 12-lead ECG before treatment initation might already identify some patients with non-interpretable QT intervals in the limb leads. However, T-wave morphology might only change with increasing QT interval prolongation, which would not be recognizable in the screening ECG. Of note, no patient in the above mentioned studies on mobile ECG QT monitoring has experienced TdP, therefore their true prognostic utility in those patients is not known. A possible solution to limited interpretability of the QT interval in the limb leads generated by mobile ECG devices could be the placement of the devices in different chest positions in order to get chest lead-like cardiograms. However, this promising approach has only been tested in a small patient population so far. ${ }^{11}$

Strengths of our study include the systematic assessment of a large number of patients with diLQTS and TdP. Limitations include the retrospective nature of our analyses of prior published cases with possible publication bias. However, due to the overall low occurrence of TdP in diLQTS, a prospective study would be unfeasible due to a large amount of participants needed. Furthermore, our study did not include a control group.

In conclusion, our results point out the limits of QT interval measurement using only the limb leads in patients with diLQTS experiencing TdP due to different T-wave morphologies in the limb and chest leads. Patients with diLQTS and high risk for TdP should probably undergo limb and chest lead ECG screening.

\section{References}

1. Roden DM. Drug-induced prolongation of the QT interval. N Engl J Med . 2004;350:1013-1022.

2. Schwartz PJ, Woosley RL. Predicting the Unpredictable: Drug-Induced QT Prolongation and Torsades de Pointes. J Am Coll Cardiol . 2016;67:1639-1650.

3. Heist E. Kevin, Ruskin Jeremy N. Drug-Induced Arrhythmia.Circulation . 2010;122:1426-1435.

4. Zhu N, Zhang D, Wang W, Li X, Yang B, Song J, Zhao X, Huang B, Shi W, Lu R, Niu P, Zhan F, Ma X, Wang D, Xu W, Wu G, Gao GF, Tan W, China Novel Coronavirus Investigating and Research Team. A Novel Coronavirus from Patients with Pneumonia in China, 2019. N Engl J Med . 2020;382:727-733.

5. Sanders JM, Monogue ML, Jodlowski TZ, Cutrell JB. Pharmacologic Treatments for Coronavirus Disease 2019 (COVID-19): A Review.JAMA [Internet]. 2020 [cited 2020 Apr 21];Available from: https://jamanetwork.com/journals/jama/fullarticle/2764727

6. Roden Dan M., Harrington Robert A., Poppas Athena, Russo Andrea M. Considerations for Drug Interactions on QTc in Exploratory COVID-19 (Coronavirus Disease 2019) Treatment. Circulation [Internet]. [cited 2020 Apr 17];0. Available from: https://www.ahajournals.org/doi/10.1161/CIRCULATIONAHA.120.047521

7. Sacher F, Fauchier L, Boveda S, de Chillou C, Defaye P, Deharo JC, Gandjbakhch E, Probst V, Cohen A, Leclercq C, Working Group of Pacing, Electrophysiology of the French Society of Cardiology, the Board of the French Society of Cardiology. Use of drugs with potential cardiac effect in the setting of SARS-CoV-2 infection. Arch Cardiovasc Dis . 2020;

8. Castelletti S, Dagradi F, Goulene K, Danza AI, Baldi E, Stramba-Badiale M, Schwartz PJ. A wearable remote monitoring system for the identification of subjects with a prolonged QT interval or at risk for drug-induced long QT syndrome. Int J Cardiol . 2018;266:89-94.

9. Garabelli P, Stavrakis S, Albert M, Koomson E, Parwani P, Chohan J, Smith L, Albert D, Xie R, Xie Q, Reynolds D, Po S. Comparison of QT Interval Readings in Normal Sinus Rhythm Between a Smartphone 
Heart Monitor and a 12-Lead ECG for Healthy Volunteers and Inpatients Receiving Sotalol or Dofetilide. $J$ Cardiovasc Electrophysiol . 2016;27:827-832.

10. Malone D, Gallo T, Beck J, Clark D. Feasibility of measuring QT intervals with a portable device. $A m$ J Health-Syst Pharm AJHP Off J Am Soc Health-Syst Pharm . 2017;74:1850-1851.

11. Cheung CC, Davies B, Gibbs K, Laksman ZW, Krahn AD. Multi-lead QT Screening is Necessary for QT Measurement: Implications for Management of Patients in the COVID19 Era. JACC Clin Electrophysiol[Internet]. 2020 [cited 2020 Apr 21];Available from: http://electrophysiology.onlinejacc.org/content/early/2020/04/06/j.jacep.2020.04.001

12. Vicente Jose, Johannesen Lars, Mason Jay W., Crumb William J., Pueyo Esther, Stockbridge Norman, Strauss David G. Comprehensive T wave Morphology Assessment in a Randomized Clinical Study of Dofetilide, Quinidine, Ranolazine, and Verapamil. J Am Heart Assoc . 4:e001615.

13. Haberman ZC, Jahn RT, Bose R, Tun H, Shinbane JS, Doshi RN, Chang PM, Saxon LA. Wireless Smartphone ECG Enables Large-Scale Screening in Diverse Populations. J Cardiovasc Electrophysiol . $2015 ; 26: 520-526$.

14. Haverkamp HT, Fosse SO, Schuster P. Accuracy and usability of single-lead ECG from smartphones A clinical study. Indian Pacing Electrophysiol J . 2019;19:145-149.

\section{Figure legends}

Figure 1 Reported drugs with potential for QT interval prolongation that patients were taking stratified by drug class. Number in the brackets denotes number of individual drugs. AAD = anti-arrhythmic drugs; $\mathrm{DPM}=$ diphenhydramine; $\mathrm{MCP}=$ metoclopramide.

Figure 2 Bland Altman plots comparing mean QTc intervals of the limb and chest leads. Horizontal line indicates mean difference.

Figure 3 T-wave morphologies in each individual lead and cumulative for limb and chest leads. Number In the brackets denotes percentages.

Figure 4 12-lead ECG showing different T-wave morphologies over individual leads in the same patient.

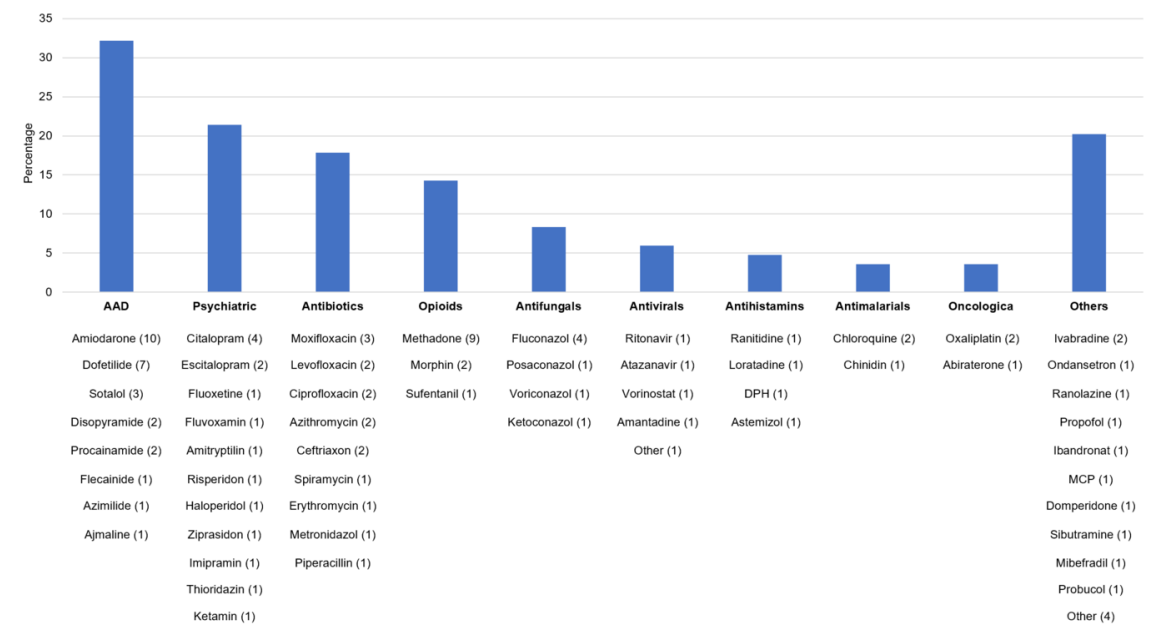

\section{Figure 1}




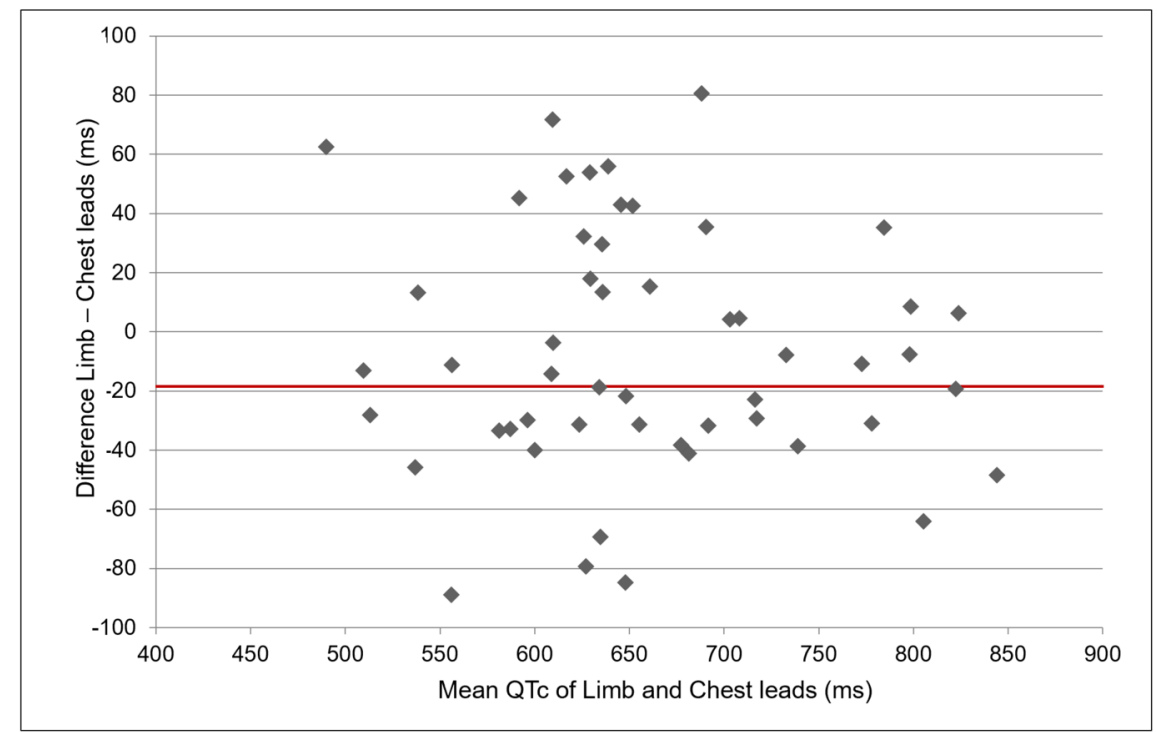

Figure 2

Figure 3

\begin{tabular}{|c|c|c|c|c|c|c|c|}
\hline & & \multicolumn{6}{|c|}{ T-wave Morphology } \\
\hline & $\mathrm{n}$ & Flat & Broad & Notched & Late peak & Biphasic & Inverted \\
\hline Limb & 469 & $239(51.0)$ & $67(14.3)$ & $21(4.5)$ & $59(12.6)$ & $25(5.3)$ & $58(12.4)$ \\
\hline I & 78 & $45(57.7)$ & $8(10.3)$ & $1(1.3)$ & $12(15.4)$ & $3(3.8)$ & $9(11.5)$ \\
\hline II & 83 & $33(39.8)$ & $16(19.3)$ & $3(3.6)$ & $13(15.7)$ & $5(6.0)$ & $13(15.7)$ \\
\hline III & 77 & $41(53.2)$ & $10(13.0)$ & $1(1.3)$ & $13(16.9)$ & $5(6.5)$ & $7(9.1)$ \\
\hline aVR & 77 & $37(48.1)$ & $14(18.2)$ & 15 (19.5) & $0(0)$ & $3(3.9)$ & $8(10.4)$ \\
\hline $\mathrm{aVL}$ & 77 & 49 (63.6) & $5(6.5)$ & $1(1.3)$ & $11(14.3)$ & $3(3.9)$ & $8(10.4)$ \\
\hline aVF & 77 & $34(44.2)$ & $14(18.2)$ & $0(0)$ & $10(13)$ & $6(7.8)$ & $13(16.9)$ \\
\hline Chest & 453 & 89 (19.6) & $67(14.8)$ & $34(7.5)$ & $33(7.3)$ & $69(15.2)$ & 161 (35.5) \\
\hline V1 & 76 & $29(38.2)$ & $6(7.9)$ & $1(1.3)$ & $5(6.6)$ & $10(13.2)$ & 25 (32.9) \\
\hline V2 & 76 & $12(15.8)$ & $11(14.5)$ & $6(7.9)$ & $4(5.3)$ & $17(22.4)$ & $26(34.2)$ \\
\hline V3 & 76 & $6(7.9)$ & $11(14.5)$ & $7(9.2)$ & $5(6.6)$ & $15(19.7)$ & $32(42.1)$ \\
\hline V4 & 74 & $8(10.8)$ & $12(16.2)$ & $8(10.8)$ & $5(6.8)$ & $11(14.9)$ & $30(40.5)$ \\
\hline V5 & 76 & $14(18.4)$ & $12(15.8)$ & $8(10.5)$ & $6(7.9)$ & $8(10.5)$ & $28(36.8)$ \\
\hline V6 & 75 & $20(26.7)$ & $15(20.0)$ & $4(5.3)$ & $8(10.7)$ & $8(10.7)$ & $20(26.7)$ \\
\hline
\end{tabular}


Figure 4

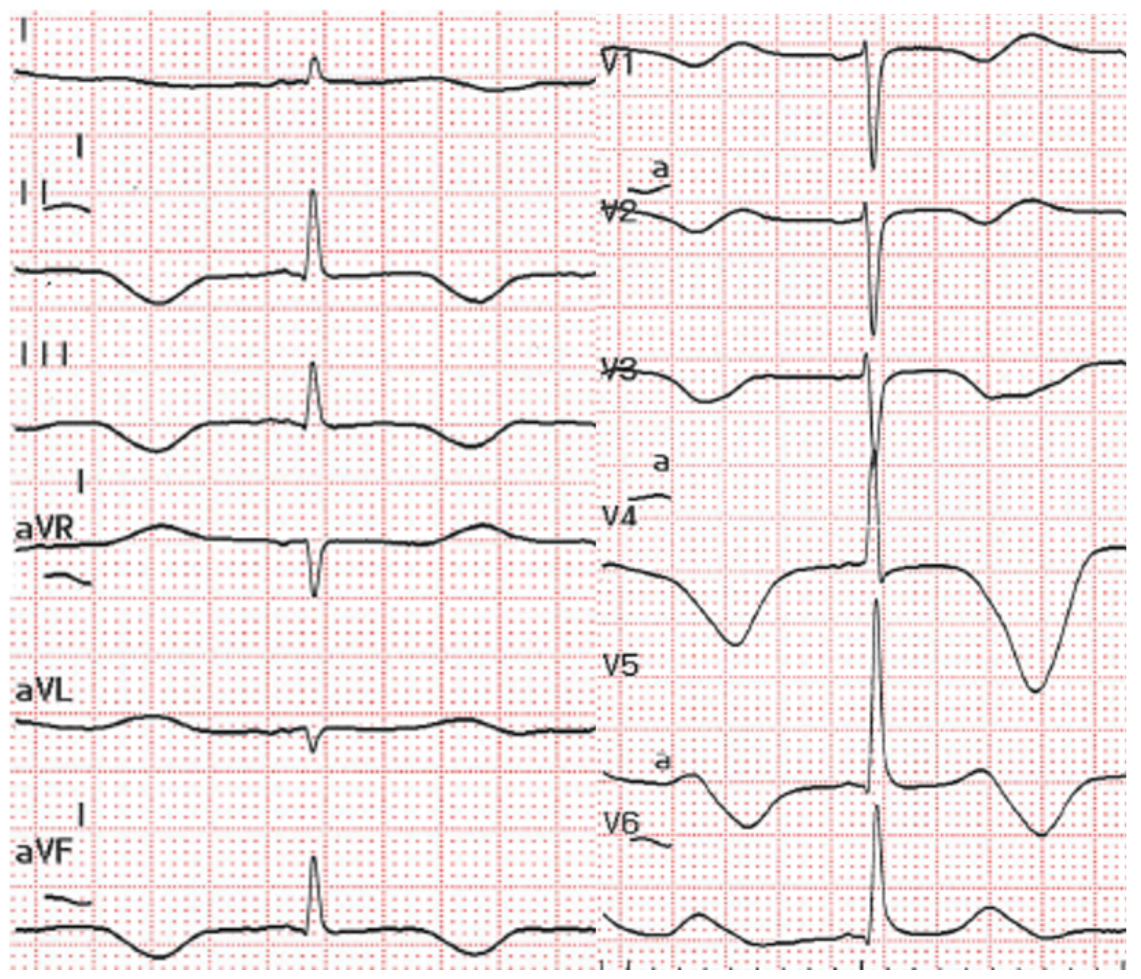

